\title{
AN ANALYSIS OF NETWORK VIRTUALISATION
}

\author{
Swapnil Raj
}

SOEIT, Sanskriti University, Mathura, Uttar Pradesh, India

\begin{tabular}{|c|c|c|c|}
\hline Journal & \multicolumn{3}{|c|}{$\begin{array}{l}\text { Samvakti Journal of Research in Information Technology } \\
\text { https://www.sjrit.samvaktijournals.com } \\
\text { Volume } 2 \text { Year of Volume } 2021 \text { Page No : } 141 \text { - } 148\end{array}$} \\
\hline Discipline & \multicolumn{3}{|l|}{ Networking } \\
\hline Conference & \multicolumn{3}{|c|}{$\begin{array}{l}\text { A virtual international conference on redefining and transforming the role of higher } \\
\text { education in sustainable development }\end{array}$} \\
\hline Conference & \multicolumn{3}{|c|}{ Start Date: September 30, 2021} \\
\hline Dates & \multicolumn{3}{|c|}{ End Date : September 30, 2021} \\
\hline Institute Name & \multicolumn{3}{|c|}{$\begin{array}{l}\text { JAIN (Deemed-to-be University) in association with Council for Industrial } \\
\text { Innovation and Research }\end{array}$} \\
\hline $\begin{array}{l}\text { Date Received } \\
\text { ID } \\
\text { Dol }\end{array}$ & $\begin{array}{l}\text { November } 21,2021 \\
2021.02 .27 \\
10.46402 / 2021.02 .27\end{array}$ & $\begin{array}{l}\text { Publication Date } \\
\text { Paper Type } \\
\text { Dol URL }\end{array}$ & $\begin{array}{l}\text { December } 11,2021 \\
\text { Conference Paper } \\
\text { https://dx.doi.org/10.46402/2021.02.27 }\end{array}$ \\
\hline
\end{tabular}

\section{ABSTRACT}

Changes to existing Internet is now restricted to modest increasing upgrades due to the presence of numerous parties with competing objectives and rules; adoption of any novel, fundamentally dissimilar technology is almost unmanageable. Network virtualization is suggested as differentiating feature of coming inter-networking model to ward against this ossification permanently. Network virtualization offers flexibility, encourages variety, and promises security and improved manageability by enabling various dissimilar network designs to coexist in same external substrate. In the article, author suggest network virtualization prototype along with set of core plan objectives, review history and current status related to network virtualization, and explore the upcoming difficulties which should be solved in order to achieve successful model of network virtualization.

KEYWORDS: Information, Internet, Network, Technology, Virtualisation.

\section{INTRODUCTION}

The Internet has been a huge success in terms of influencing how humans access and share data in contemporary world. Internet architecture has proved its value over the last three decades by enabling a broad range of distributed applications and network technologies. Its popularity, in the other hand, has become the most significant barrier to its further expansion. Because of multiple-supplier design, 
introducing novel structure or altering an prevailing one entails consensus amongst contending parties. As consequence, modifications in architecture of Internet have been restricted to minor tweaks rather than major overhauls including the introduction and organization of novel technologies of network ${ }^{[1]}$.

Virtualization has become a prominent idea in information and communications technology (ICT) industry in a variety of domains, including virtual data centers, virtual machines, , virtual storage access networks, and virtual memory. It entails resource generalisation and allocation among many users. Because of higher consumption of hardware, de coupled functions as of structure, faster relocation to novel services and items, and versatile administration, the total price of apparatus and supervision may substantially lowered with virtualization.

Although architectural classicists see network virtualization as method of assessing novel designs, the pluralist method views virtualization in form of basic feature of architecture. Agreeing to these, network virtualization may mitigate present Internet's ossification pressures while also stimulating innovation by letting diverse network designs to co-occur in same external substrate. Partitioning of policy and machinery is properly-verified concept in computer collected works for introducing variety ${ }^{[2]}$.

For virtualizing networks, a similar method has been proposed. Traditional ISPs' roles have distributed into 2 categories in this case: infrastructure providers that cope up physical infrastructure and service suppliers that build virtual networks through pooling resources as of numerous arrangement suppliers and providing end-to-end facilities to end users. These kind of environment would encourage the implementation of various co-occuring diverse network designs which aren't constrained by current Internet's fundamental constraints. This article covers network virtualization's history and current state-of-the-art, as well as important problems that should be investigated further in the future ${ }^{[3]}$.

Virtualization has been a prominent idea in ICT industry in a variety of domains, including memory which is virtual [1], machines which are virtual [2], storage which have virtual access network [3], and data centers which are virtual [4]. It entails resource encapsulation and distribution amongst several users. Because of greater hardware consumption, decoupled functionality from architecture, simpler transfer to updated services and goods, and versatile administration, the entire price of the goods and maintenance may be greatly lowered with virtualization.

\section{Historical Perspective:}

Multiple coexisting networks isn't necessarily a novel idea. It has already appeared in the networking literature in various forms. We'll look at three of them in this part, all of which are strongly linked to the idea of network virtualization. A VPN, also acknowledged as a virtual private network, is customized virtual network which links many dispersed locations over tunnels across mutual or open networks. Another kind of network virtualization are an overlay network that is usually executed at application 
layer but there are also executions at lower levels of stack of network. It's widely used on the Internet as rudimentary whereas efficient method for deploying new features and updates. Programmable and Active networks, whereas are idea whichs allows network components to be customized depending on the needs of service providers ${ }^{[4]}$.

\section{Virtual Private Network (VPN):}

VPN is devoted system for communications for multiple businesses which is spread to several locations and linked with tunnels across mutual or public communication networks such as Internet. When each of the site in VPN belong to identical company, VPN is referred to corporate Intranet. It is recognized as Extranet if sites are operated by separate businesses. The majority of VPNs in use today are intranets that link geographically dispersed offices of big corporations. 1 or additional Customer Edge (CE) devices must be present at each VPN location (example, hosts or routers). Every CE device is connected to 1 or additional Provider Edge (PE) routers through an attachment circuit. ' $P$ ' routers are routers in SP's network which don't connect to devices of CE. Provider-supplied VPN (PPVPN) is a kind of VPN that is controlled and provisioned with VPN service provider (SP). Depending upon protocol utilized in VPN data layer, PPVPN technologies are divided into 3 classifications ${ }^{[5]}$ :

\section{a. Layer 3 PPVPN:}

The implementation of protocols of layer 3 ( IP or MPLS) in common substructure of network (backbone of VPN) for transporting files among dispersed CEs distinguishes Layer 3 VPN (L3VPN) [9, 10]. L3VPNs may be divided into 2 types: CE-based VPNs and PE-based VPNs. These shared service provider network has no awareness of the customer VPN in CE-based VPN method. CE apparatuses construct, maintain, and dismantle channels. SP network is totally ignorant of the VPN, and packages are treated as regular IP packets. Tunneling requires 3 distinct protocols ${ }^{[6]}$ :

I. The SP network uses a carrier protocol (such as IP) to transport VPN packages.

II. Encapsulating protocol, which wraps the raw data in a protective layer. It may be anything from a basic wrapper protocol to a secure protocol.

III. The initial data in consumer networks is the Passenger Protocol.

Passenger packets are encapsulated and routed through carrier networks by sender CE devices; when these encapsulated packages reach to $C E$ devices of receiver at tunnels end, these have to be removed and real packages is inserted in networks of receiver side On the other hand, with PE-based L3VPNs, entire states is preserved in devices of PE, and linked device of $C E$ might act if it was associated to isolated network. In such instance, PE devices recognize that some traffic is VPN related and handle it appropriately. Virtual Router PPVPN refers to a PE-based VPN that conserves full logical router through exclusive progressing table and routing protocol configured for individual VPN. BGP/MPLS IP VPN is when single instance of BGP is distributed among across VPNs by means of distinct promoting environments and promoting tables for every one of these. In this instance, characteristics are used to determine the VPN context of route advertising ${ }^{[6]}$. 


\section{b. Layer 2 of VPN:}

It (L2VPNs) transmit frames of Layer 2 among participating sites (usually Ethernet, but sometimes Frame Relay and ATM). L2VPN has the benefit of being agnostic to higherlevel protocols and being simpler. However, it has no control plane for governing ability of reach through VPN, which is a drawback. Virtual Private Wire Service (VPWS) and Virtual Private LAN Service (VPLS) are two types of services of Layer 2 VPN which service supplier may deliver to client (VPLS). There's also the option of a LAN-like IPonly service (IPLS). A VPN service which provides point-to-point L2 service is known as VPWS. A VPLS is L2 service which is point-to-multipoint which replicates LAN functionality through a wide area network. IPLS are identical with VPLS with the exception that devices of $C E$ is routers or hosts rather than switch, and IP packages (IPv4 or IPv6) is transported.

\section{c. Layer 1 of VPN:}

This (L1VPN) architecture arose after necessity for expanding L2/L3 packageswitching of VPN ideas for forward-thinking domains in circuit-switching, accompanied by fast advancements in coming-group SONET/SDH and optical swapping, as well as control related to GMPLS. It allows for provisioning of numerous virtual clientprovisioned transport networks above a shared layer 1 core substructure. Primary distinction amongst L1VPNs and L3 or L2 VPNs is data plane connection don't imply connectivity of control plane in L1VPNs.

However, for services of L1VPN provided via control plane, CE-PE control plane connection are needed, and CE-CE data plane connectivity is sustained through indicating methods depending upon this connectivity of control plane. L1VPN's primary feature is that it offers a multi-service backbone via which clients may provide their personal facilities alongwith payloads related to any layer. This guarantees every single of network of service has its own space address, view of resource of layer 1 , rules, and is completely isolated with the other networks which are virtual. VPWS and VPLS are two kinds of L1VPN (VPLS). VPWS is a service which is point-to-point, whereas VPLS may considered a service which is point-to-multipoint.

\section{Programmable and active network:}

The requirement to quickly build, implement, and managing of new services in reaction to customer needs was a major driving force behind the development of programmable networks research. A key need for enabling on demand services is separating control software and communications hardware. If such separation is in place, software may be designed to perform required functions regardless of the underlying hardware. The programmable networking community is debating how to accomplish this separation. The authors provide a review of programmable networks as well as a general programmable networking paradigm, in which programmability is accomplished by 
adding computing inside network and by increasing the quantity and breadth of calculation done in current switches and routers ${ }^{[7]}$.

A programmable network is made up of computing and communication models that enable a network designer to programme specific layers through management, transport and control, planes. A collection of distributed node kernels and environment of network programming are used to implement the computing paradigm. Node kernel is most basic level with programming ability, offering limited range of interfaces of node for manipulating node states. Network programming environments enable the vibrant execution of network services and protocols, allowing for the creation of networks. They provide network designers with a set of services and open interfaces for programming bespoke network architecture. On how to really execute this idea, two schools of thought emerged: one from the telecoms industry and the other from the IP community of networks.

\section{a. Open Signalling Approach (Opensig):}

It offers a telecommunications-oriented approach to the issue, emphasizing the difference between the control, transport, and administration planes which make up programmable networks, as well as QoS provides assurance for newly developed facilities. It advocates for modelling communiqué hardware with the use of set of open network interfaces which are programmable, allowing third-party software suppliers free access to switches and routers. It provides abstraction layer that allows external devices with network for behaving as shared objects of computing with properlyexplained interfaces which are open that enable package suppliers for modifying conditions of network.

\section{b. Active Networks Approach (AN):}

Within the confines of current networks, the networks community which are active supports vigorous arrangement of novel amenities at execution time. Such network is active in a manner that switch or router may conduct personalised calculations and modifications depending on packet contents. Instead of customizing services related to network at the package relocate granularity with control plane which is programmable, the Networks which are Active approach allows for customization at the packet transport granularity; as a result, it provides more tractability than the Opensig method at charge of more complicated model of programming. Over the years, several degrees of programmability have been proposed. At one extreme of the spectrum, ANTS [26] provides a machine model which is Turing-complete at active router, allowing any for to running any novel code. DAN, on another hand, only enables user to invoke functions that are previously set up at a certain node. A researcher divided the suggested structural design into three categories depending on control granularity, language expressive capacity and state fullness. 


\section{Overlay Networks:}

It is a virtual computer network that overlays the external topology of other network to produce a virtual topology. Virtual connections, which may correspond to a route, connect nodes in an overlap network, which are linked by numerous external contacts in primary network. These aren't limited by geography, contribution too is entirely optional. Overlays are usually low-cost since members contribute their resources to the network on a voluntary basis. Furthermore, in contrast to any other network, they are versatile and adaptive to changes, as well as being simple to install. As an outcome, these networks have been used from very long to deliver new Internet attributes and repairs.

In recent years, a slew of overlap strategies have been suggested to resolve a variety of concerns, including making sure Internet routing enactment and accessibility, allowing multicasting, provided QoS guarantees, shielding against denial of service (DoS) attacks, for circulation of content, distribution of file, and infact storage systems. According to the Detour research, re-routing packets via virtual tunnels may frequently outperform the straight Internet route in terms of loss, throughput, and latency. The Resilient Overlay Network (RON) research demonstrated that an overlap system that conducts measurements of its own network may offer rapid failure recovery as well as reduced expectancy and loss rates even over small timescales. By separating the sending and receiving actions, the overlap-dependent Internet Indirection Infrastructure (i3) promises for shortening of network service implementation and administration. Sources deliver packages to a reasonable identifier in i3, while receivers show interest in packages sent to identifier. This extra layer of abstraction provides for more freedom in mobility of node, as well as service placement and deployment. In all 3 instances, the Internet's routing performance is improved by layering an overlap on highest of current directing sub strate.

Routing as a Service (RaaS) presents Routing Service Providers (RSPs) which is third-party and purchase virtual connections through several ASes to connect a group of virtual routers. Hosts that want personalized routes agreement with RSP, which then creates a suitable end-to-end overlap route alongside their virtual connections depending on the topology's universal perspective. This concept of bringing in thirdparty suppliers is very appealing. OverQoS is a method for establishing overlay connections with guaranteed loss and latency. The Service Overlay Network (SON) is intended to offer value-added Internet services using an overlay approach. A SON may buy bandwidth from several ISPs with certain QoS assurances to create a logical end-to-end facility supply overlay.

\section{DISCUSSION}

The overall expenses of constructing and managing a wireless network might be significantly reduced thanks to wireless network virtualization, which enables for the generalization and exchange of radio spectrum design and assets. By isolating a 
segment of the system, wireless network virtualization could simplify things to migrate to updated goods or techniques. Regardless of the potency of wireless network virtualization, innumerable major research hurdles must also be acknowledged before widespread deployment, including exclusion, tracking alerting, asset immersion and allotment, packet transmission, network management and implementation, and confidentiality, as well as non-technical troubles such as governance regulations. In this article, we provide a brief summary of a few of the prior attempts to achieve wireless network virtualization, as well as discuss pertinent research issues and roadblocks. Wireless network virtualization requires an overview, motivations, organization, performance measurements, enabling technologies, and challenges. Finally, author address the ramifications of wireless network virtualization on a larger scale.

\section{CONCLUSION}

Among the recent trends of virtualizing virtually each element of computing, including operating systems, systems for storage, servers, and infact huge centers of data (example, cloud computing), network virtualization is one which stands out. On the one hand, network which is virtualized is required for linking with whole other machines which are virtualized and provide every virtual entity with a full resemblance to their inborn equivalents. Whereas on other hand, after decades of fast development, Internet and networking in general have reached a stalemate. The majority of academics now believe that a redesign is a requirement rather than a luxury. In this situation, network virtualization may play a key role in promoting innovation, providing flexibility, and introducing heterogeneity. This discovery has spawned a slew of initiatives across the globe which are either indirectly or directly linked to network virtualization. However environment of network virtualization must be realized in accordance with its features and plan objectives. However nevertheless this criteria will guarantee an exposed, adaptable, and diverse environment for networking, these will not be simple to meet. We urge further study into the difficulties raised in the paper and look ahead to encouraged efforts by scholars in this area to find answers to the open research challenges. 


\section{REFERENCES:}

[1] N. M. M. K. Chowdhury and R. Boutaba, "A survey of network virtualization," Comput. Networks, 2010, doi: 10.1016/j.comnet.2009.10.017.

[2] A. Blenk, A. Basta, M. Reisslein, and W. Kellerer, "Survey on network virtualization hypervisors for software defined networking," IEEE Communications Surveys and Tutorials. 2016, doi: 10.1109/COMST.2015.2489183.

[3] Z. Khalid, N. Fisal, and M. Rozaini, "A survey of middleware for sensor and network virtualization," Sensors (Switzerland). 2014, doi: 10.3390/s141224046.

[4] A. Belbekkouche, M. M. Hasan, and A. Karmouch, "Resource discovery and allocation in network virtualization," IEEE Commun. Surv. Tutorials, 2012, doi: 10.1109/SURV.2011.122811.00060.

[5] Y. Wang, P. Chau, and F. Chen, "Towards a secured network virtualization," Comput. Networks, 2016, doi: 10.1016/j.comnet.2016.04.023.

[6] Y. Guo, H. Zhu, and L. Yang, "Service-oriented network virtualization architecture for Internet of Things," China Commun., 2016, doi: 10.1109/CC.2016.7582308.

[7] M. F. Bari et al., "Data center network virtualization: A survey," IEEE Commun. Surv. Tutorials, 2013, doi: 10.1109/SURV.2012.090512.00043. 